\title{
ALGUNAS CONEXIONES \\ DE LA TEORÍA KANTIANA DEL DERECHO
}

\author{
Felipe MARTÍNEZ MARZOA \\ Universidad Central de Barcelona
}

RESUMEN: Se recuerdan brevemente algunas precisiones interpretativas ya anteriormente defendidas acerca de la concepción kantiana de lo juridico y, sobre esta base, se establece la conexión de dicha concepción con dos posiciones modernas referentes a lo político. Una de ellas es el uso que hace Marx del concepto "república democrática" como, a la vez, expresión de la sociedad civil y figura juridico-política de la revolución. La otra es lo que en el terreno político puede seguirse del proyecto nietzscheano de la asunción del nibilismo.

Está fuera de toda duda que el concepto del complejo Estado-derecho-leyes comporta la capacidad (en último término física) de imponer. Nadie hablaría de Estado (ni de lo que en tal contexto cabe entender por derecho y leyes) si no hubiese capacidad coercitiva suficiente para que las leyes en general se cumplan $y$, lo que es lo mismo, en relación con su incumplimiento, capacidad penal suficiente para, en general, evitarlo.

Por otra parte, parece igualmente cierto que no cualquier capacidad coercitiva suficiente para hacer cumplir unas reglas es ya, por el hecho de ser tal capacidad, Estado, ni, lo que es lo mismo, el que esa situación se dé convierte por sí solo esas reglas en derecho (ni siquiera en el específico sentido en que esta palabra se emplea en contexto con el concepto de Estado). En otras palabras: parece necesario algún modo (todo lo "neutro" que se quiera) de distinguir entre el fenómeno "Estado» y el trivial secuestro de una población, e incluso parece que ese modo de distinguir debe ser independiente de si la población padece o no el síndrome de Estocolmo.

De todo esto, sin embargo, se desprende también que la nota distintiva a cuya búsqueda en ello se apunta no puede en manera alguna consistir en algo "ético" o "moral»; pues no puede en absoluto tener ese carácter algo a lo cual es 
posible obligar por la fuerza, y precisamente acabamos de decir que del complejo Estado-derecho-leyes no puede formar parte nada que no pueda, en caso necesario, ser obtenido por la fuerza.

En otras partes, el autor de estas líneas ha ido desarrollando una interpretación del conjunto de la «filosofía práctica» de Kant ${ }^{1}$ cuyo ejercicio es de especial interés en relación con la cuestión que ahora acaba de plantearse. Recordemos que, según esa interpretación, la fundamentación kantiana de la posibilidad de una autocrítica de la decisión cuyo criterio no proceda de ninguna otra parte que de la decisión misma enjuiciada (el que en la decisión misma esté incluido el autoenjuiciamiento de ella misma) se hace reconociendo como decisión no otra cosa que una función del conjunto de las posibles situaciones cognoscitivamente dadas en el conjunto de las posibles conductas materiales, función en la que sólo la función misma es decisión, mientras que tanto los elementos del conjunto de partida como los del conjunto de llegada son objetos de conocimiento. Esta determinación tiene, como allí mismo se hace ver, la importante consecuencia de que la decisión es (no por proclamación doctrinal alguna, sino tautológicamente) inaccesible a la coacción, ya que ésta no hace sino cambiar el elemento del conjunto de partida, a lo cual puede corresponder, según la misma función, un cambio de elemento del conjunto de llegada, de manera que, en efecto, la coacción nada tiene que ver con cambio alguno en la función misma, esto es, en la decisión; ésta puede, desde luego, cambiar (su contenido es contingente), pero no como consecuencia de la coacción. Este mismo análisis deja también manifiesto que la decisión tiene el carácter lógico de algo uno aplicable en principio a infinitos casos posibles, es decir, tiene la forma lógica de universal. Y así queda también entendida la posibilidad de la contradicción que necesariamente ha de poder haber en la decisión para que ésta pueda contener su propio autoenjuiciamiento; en efecto, la contradicción consiste en que cierta decisión, teniendo como tal la forma lógica de universalidad, a la vez tenga un contenido que no es compatible con esa forma, lo cual es posible porque, al ser contingente la decisión, la universalidad como forma lógica nunca es universalidad efectiva, es decir, nunca puede tratarse de, en efecto, aplicar la misma decisión a todos los casos de posible aplicación de ella misma.

${ }^{1}$ El más reciente documento al respecto (el cual, a los efectos que importan a la presente exposición, puede valer como resumen o balance de los anteriores) es mi artículo Reconsideración del concepto de ética autónoma (en "La ortiga", n. ${ }^{\circ} 33-35,2002$, pp. 35-45). 
Lo referente al autoenjuiciamiento de la decisión al que ahora nos estamos refiriendo es lo que llamamos lo «ético" o lo «moral». Dado que una misma conducta material en una misma situación cognoscitivamente dada puede responder a decisiones (a funciones) diferentes e incompatibles entre sí, el enjuiciable ético no es en sí mismo un posible objeto de descubrimiento cognoscitivo. $Y$, sin embargo, es en efecto éticamente enjuiciable, pues puede suceder que la observación de mi conducta material permita concluir que la misma no puede ser resultado de decisión alguna cuyo contenido sea compatible con la forma lógica de universalidad, y en tal caso, ciertamente, no sé cuál es mi decisión, pero sé que es éticamente incorrecta. Este es incluso el único modo de enjuiciamiento ético que podemos ejercer en concreto; es ético; enjuicia el enjuiciable ético (ciertamente sin conocerlo en sí mismo, y es perfectamente coherente el que no se lo pueda conocer en sí mismo) y lo enjuicia según el criterio ético; observa la conducta material porque ésta es, por definición, lo único que se puede conocer, pero la observa para obtener conclusiones acerca de la decisión (aunque sin conocer ésta en sí misma), y de la conducta material observa una "legalidad", pero esta palabra aquí sólo significa compatibilidad con la forma lógica de universalidad (con la forma de «ley») en el ya indicado sentido de que no sea imposible que haya algún contenido de decisión compatible con la forma lógica de universalidad generador de esa conducta material. Así, pues, todo esto no tiene todavía relación alguna con el derecho; si la tuviese, entonces sería penable toda conducta en la que pudiese detectarse incorrección ética, lo cual es un disparate sin paliativos.

Muy otro es el camino que de verdad conduce kantianamente a la noción del derecho y de lo jurídico; es un camino que excluye toda posibilidad de que de lo ético se derive algo jurídico y, una vez demostrado que de todos modos tiene que haber una esfera de lo jurídico, toma como principio de tal esfera no otra cosa que la mencionada exclusión (si se quiere: autoexclusión) del criterio ético en ella. Veámoslo con un poco más de detalle.

El reconocimiento del criterio ético pasa inevitablemente - lo vimos- por el hecho de que el enjuiciable ético (la decisión) se delimita de tal manera que resulta ser tautológicamente inmune a la coacción. Esto tiene como evidente consecuencia el que nunca una exigencia ética podrá servir de base para legitimar coacción alguna ni, por lo tanto, para distinguir entre una coacción legítima y una ilegítima. Y entonces parece que hace falta algo nuevo en la sistemática del pensamiento kantiano; pues coacción no es algo que pueda haber o no 
haber; la hay por el hecho mismo de que se existe en el mundo físico, por lo tanto bajo el principio de interdependencia; por ejemplo: donde estás tú no puedo estar yo y viceversa, y esto ya es coacción. Parece, pues, que no cabe eludir la cuestión de una posible distinción entre coacción legítima e ilegítima. Y ya hemos visto que esa distinción no puede fundamentarse en la exigencia ética. Veamos si esta misma ausencia de fundamento ético puede constituir una especie de fundamento. Tal ausencia, en efecto, reclama de toda posible coacción que ésta se abstenga de juicios morales. A efectos de posible coacción nada es "bueno" ni "malo" ni "mejor" ni "peor", no hay otra legitimidad que la de que yo pueda hacer lo que me venga en gana, y, en esto que acabo de decir, "yo" es cualquiera, de manera que lo que en verdad acabo de decir es que yo he de poder hacer todo aquello tal y bajo condiciones tales que el hecho de que yo lo haga no sea incompatible con que cualquier otro bajo las mismas condiciones pueda también, si quiere, hacerlo. Esa es la frontera entre legítimidad e ilegitimidad en materia de posible coacción, y, por lo tanto, eso es el «derecho».

Por la exposición que hasta aquí se ha hecho, o a la que se ha remitido, cierta fórmula definitoria del concepto del derecho ha quedado ubicada como parte irrenunciable del pensamiento de Kant. Será, pues, una contribución interesante al aprovechamiento de la capacidad esclarecedora que ese pensamiento tiene el reconocer en la citada fórmula definitoria del derecho algo que también a través de otros autores emerge como una pieza básica en la detección del fenómeno específicamente moderno Estado-derecho y en la definición de lo moderno mismo en general.

Marx caracterizó alguna vez ${ }^{2}$ la "república democrática" como la forma de Estado última y definitiva. Para los objetivos que en el presente trabajo nos proponemos será de interés aclarar en qué sentido lo es, lo cual comportará de paso aclarar qué tiene todo ello que ver con la tesis kantiana sobre el derecho que acabamos de presentar.

La inacabada obra de Marx "El capital»" ${ }^{3}$ es la exposición de cómo el concepto "mercancía", inicialmente asumido como concepto en sentido trivial, es

${ }^{2}$ Kritik des Gothaer Programms, MEW XIX, p. 29.

${ }^{3}$ Cf. mi libro La filosofía de 'El capital', Madrid 1983, así como mis artículos Estado y legitimidad y Estado y pólis, ambos en: M. CRUZ (comp.): Los filósofos y la política, Madrid 1999, pp. 85-115. 
decir, nota o suma de notas, es llevado por su mismo contenido (y en esto difiere de cualquier concepto trivial) a modificar su propia' naturaleza lógica en una doble manera, a saber: por una parte pasa del estatuto de nota o conjunto de notas al de "construcción" (tránsito al que está sometido también un concepto trivial, pero no cada uno de ellos por exigencia de su propio contenido, cosa que, en cambio, sí ocurre con "mercancia») y por otra parte - y esto ya sin nada en común con el caso de un concepto trivial — la construcción en cuestión pasa a ser no ya el ejercicio de una regla repetible distributivamente, sino la construcción de un singular omniabarcante, la de una estructura o sistema de todas las cosas. Lo que así se establece o construye, es decir, la estructura o sistema de todas las cosas que incluye todo lo necesario para que en efecto haya mercancía, eso es lo que en el lenguaje del propio texto y en el de un contexto bastante amplio se llama la "sociedad civil». El primer paso de la construcción consiste en la demostración conjunta de dos tesis. Una: el fenómeno mercancía, es decir, el que el cambio de unas cosas por otras sea general, implica que haya una objetividad en la determinación de en qué proporción se cambian entre sí cantidades de diversas cosas; por lo tanto, si hay intercambio general de cualesquiera tipos de cosas, entonces hay algo, llamémosle $\mathrm{S}$, tal que: el que la cantidad $\mathrm{x}$ de la mercancía A equivalga en el cambio a la cantidad y de la mercancía $B$ implica que hay la misma cantidad de $\mathrm{S}$ en la cantidad $\mathrm{x}$ de $\mathrm{A}$ que en la cantidad $\mathrm{y}$ de $\mathrm{B}$; digamos: hay algo así como una "substancia» de la que hay la misma cantidad en dos cantidades de cosas cualitativamente distintas entre sí que equivalgan la una a la otra en el cambio. Esa es una de las dos tesis; la otra es que la substancia en cuestión no puede ser ninguna característica física de las cosas ni, por lo tanto, magnitud física alguna. El que la demostración conjunta de ambas tesis constituya el desencadenamiento del proceso de construcción significa que éste empieza con el reconocimiento de una "objetividad no física" y que el propio proceso no será sino desarrollar en qué consiste y qué comporta esa objetividad no física, la cual podemos, pues, identificar con lo que venimos llamando la sociedad civil. Lo que ahora nos interesa destacar es que a la construcción es inherente el que la objetividad no física tiene lugar precisamente y sólo en el sentido de que las relaciones de cambio entre las cosas la implican, es decir, no tiene lugar en sí misma y por separado; la substancia implicada en que $\mathrm{xA}$ equivalga a $\mathrm{yB}$, ambos a $\mathrm{zC}$, etcetera, tiene como formas de manifestación $\mathrm{xA}$ en cuanto intercambiable con $\mathrm{yB}, \mathrm{zC}$, etcetera, $\mathrm{yB}$ en cuanto intercambiable con $\mathrm{xA}, \mathrm{zC}$, etcetera, etcetera; no tiene una comparecencia aparte; comparece en esas formas de manifestación, no en sí misma. Esto equivale a decir: la substancia, la objetividad no física, comparece en nuestro análisis y no en su propio funcionamiento; la sociedad civil comparece como tal en una teoría de la sociedad civil, no en la sociedad 
civil misma, en su tener lugar. Con esto se fundamenta ya desde el primer paso la distinción entre la teoría de la sociedad civil y la implícita autointerpretación o autoconciencia de la propia sociedad civil; digo «implícita" porque se trata de su necesaria autoconciencia o autointerpretación, con independencia de que se la formule o no. Ejemplo de la distinción que acabo de mencionar entre, por una parte, la sociedad civil (la cual comparece en la teoría de la sociedad civil) y, por otra parte, la propia autointerpretación o autoconciencia de la sociedad civil: la teoría de la sociedad civil reconocerá que el hecho de que las cosas físicamente diversas hayan de poder a la vez servir como formas de manifiestación de cantidades de una misma magnitud no física (S) quizá imponga ciertas condiciones incluso relativas a cómo haya de entenderse el ser físico de las cosas (y ahí está en efecto el sentido específicamente moderno del concepto de lo físico, etcetera); por su parte, la propia autointerpretación de la sociedad civil, puesto que no reconoce $S$, sino sólo la relación de cambio entre las cosas, habrá asumido desde luego las condiciones a las que acabo de referirme, pero sólo en el sentido de que entenderá el ser mismo (ser físico) de las cosas de acuerdo con esas condiciones, sin reconocerlas como tales y desde luego sin referirlas a ningún $S$. Otro ejemplo tiene un papel más central en nuestra presente exposición: el fenómeno mercancía, en cuanto que sólo es entendible como carácter de toda cosa, comporta la alienabilidad de todos los contenidos; es mío precisamente aquello de lo que puedo desprenderme; no estoy vinculado a nada, no estoy obligado a nada; puedo hacer lo que me dé la gana, hasta tal punto que incluso el "yo" implícitamente mencionado en esta fórmula, por su mismo carácter de desvinculado, es cualquiera y, por lo tanto, el despliegue semántico de ese "puedo hacer lo que me dé la gana" es "puedo hacer cualquier cosa tal o bajo condiciones tales que el que yo la haga no sea incompatible con que cualquier otro bajo las mismas condiciones pueda también, si quiere, hacerlan; y eso es lo que llamamos el derecho, y de ello deriva, por cierto, el concepto «república democrática».

Hemos llegado así, siguiendo a Marx, a una noción del derecho enteramente idéntica con la que habíamos establecido leyendo a Kant. Para los fines de nuestra presente exposición, es importante el que se entienda cierto doble papel que en Marx desempeña esa noción, lo cual nos obliga a introducir todavía a continuación alguna otra consideración de origen marxiano.

La distinción que la teoría de la sociedad civil posee entre la propia sociedad civil y una autoconciencia implícita de ésta obliga a la teoría a entender la sociedad civil misma como meramente la estructura o la "objetividad no física", consistente en sí misma y de la cual, por lo tanto, es distinta incluso su propia auto- 
conciencia; en otras palabras: la sociedad civil acontece, es o funciona, no "dice" ni "piensa", o, si se prefiere decirlo así, ella, en cuanto que "dice" o "piensa», ya no es la sociedad civil, sino alguna otra cosa. En cuanto al carácter de esta otra cosa, nos limitaremos a algo que de alguna manera ya ha quedado dicho: por una parte, no se reconoce un $S$, sino que las implicaciones de $S$ son atribuidas simplemente al ser mismo de las cosas; por otra parte, lo que se autointerpreta en esa autointerpretación sólo puede ser - así lo ha puesto de manifiesto la teoría - el carácter o condición de toda cosa, por lo tanto ese "ser de las cosas" no podrá ser sino el de toda cosa en cuanto tal, la noción misma de validez, el criterio de qué hay y qué no hay, qué vale y qué no vale. Estas consideraciones son también relevantes a la hora de determinar desde dónde, con qué criterios o ante qué tribunal podría quizá la sociedad civil —la sociedad moderna - ser puesta a prueba. El acudir a algún tipo de instancias externas dejaría por definición sin posible respuesta la cuestión de por qué habrían de ser válidas esas instancias para juzgar algo a lo que son externas; más aun: quizá no pueda entenderse cómo puede haber instancias externas o qué o quién puede estar fuera de algo que sólo puede tener lugar como el sistema de todas las cosas. En cambio, la distinción entre la sociedad civil y su propia implícita autoconciencia, distinción que incluye el que esa autoconciencia no es para ella misma autoconciencia de la sociedad civil, sino reconocimiento de criterios válidos en sí mismos, puede ser la base para que la sociedad civil resulte confrontada con el hecho de que ella misma, quizá, no es capaz de cumplir en efecto esos criterios.

El hecho de que ahora vayamos a referirnos a Nietzsche requiere sin duda un trámite previo, pues el nombre de ese pensador en contexto con la presente cuestión puede invitar a un fácil juego de amontonamiento de citas también demasiado fáciles; todo el mundo recordará las diatribas de Nietzsche contra la "democracia» y contra la "igualdad" que supuestamente iría con ella. Pues bien, lo que hay de igualdad en el concepto del derecho que hemos formulado ( $\mathrm{y}$ en el sistema de las garantías y las libertades, que es despliegue de ese concepto) no tiene nada en común con esa «igualdad» que Nietzsche ataca. Quien confunda la una con la otra ignora el viejo argumento "erístico" según el cual, puesto que la diferencia de $A$ con respecto a $B$ es a la vez la de $B$ con respecto a $A$, ambos, $A$ y $B$, son iguales precisamente en cuanto que son diferentes, y no otra cosa que la diferencia es la igualdad. Aplicado a nuestro presente asunto, ese argumento dice que la única igualdad que puede haber es la garantía de la posibilidad de la diferencia, y que la otra "igualdad", la que consistiría en precisamente ser iguales, es un pseudoconcepto, algo que ni siquiera resiste el mero análisis semánti- 
co. En la hipótesis de un estricto cumplimiento del concepto del derecho que aquí hemos obtenido, seríamos más desiguales que nunca, porque ese igual derecho es precisamente el derecho a hacer usos máximamente desiguales de él. $\mathrm{O}$ dicho de otra manera: la desaparición de la udesigualdad" ficticia y preestablecida no es sino la base para el despliegue de la desigualdad real.

El tipo de observación que acabamos de hacer es incluso susceptible de alguna generalización. Con independencia de los significados modernos que cierto pseudogriego convencional atribuya a los componentes de las palabras, lo cierto es que ni "democracia" ni ningún otro término pueden significar cosa alguna parecida a que algo llamado "el pueblo" tenga algo llamado "el poder", porque no hay ningún análisis de la cuestión del poder en el que alguna entidad del análisis se llame el pueblo. Cuando alguien apoya su actuación en discursos en los que se produce como si en verdad creyese que hay una cosa llamada «el pueblo» y que esa cosa debe tener "el poder", decimos que estamos ante un "populista", y con ello queremos decir algo muy distinto de (y probablemente incompatible con) que se trate de un demócrata. Independientemente de cómo se explique su formación, la palabra "democracia" o "república democrática" ha llegado a significar en la teoría moderna algo bastante consistente, a saber, un sistema de garantías de derechos y libertades cuyos componentes (derechos y libertades que se garantizan) están en efecto vinculados entre sí de manera que es imposible suprimir o restringir alguno de ellos sin que queden a la vez suprimidos o restringidos los demás, e incluso imposible suprimir o restringir para alguien sin que ello comporte supresión o restricción para todos, en otras palabras: un sistema que sólo puede ser válido siéndolo para todos y para todos los casos, sistema cuyos componentes se generan precisamente a partir de la exigencia de que ellos sean aquellos tales que puedan ser consistentemente reconocidos para todos y para todos los casos; fórmula manifiestamente sinónima de la que por dos veces, siguiendo en una a Kant y en la otra a Marx, hemos adoptado como concepto general del derecho.

En Nietzsche, como es sabido, la palabra "nihilismo" designa el estado de cosas pendiente de asunción. Las raíces de que el estado de cosas sea ese tienen que ver con el hecho mismo de que tenga lugar en general lo que llamamos «el estado de cosas». En efecto, el que de alguna manera comparezca o acontezca el juego mismo como tal, esto es, se vuelva relevante no esta o aquella cosa, sino en qué consiste el que haya cosa, comporta una cierta pérdida o detención de eso mismo que así acontece. Podemos incluso hacer un poco más detallado este 
esquematismo: la comparecencia de aquello en lo que consiste que haya cosa comporta que ello comparece como tal o cual, esto es, que hay un cierto «ser (ser cosa) consiste en ...", y esto significa algo así como criterio(s) del "ser cosa»; entonces el que algo tenga la condición de cosa o de ente ya no consiste en la inmediatez, sino en una mediación; lo en verdad ente es un "trans-»; eso de "lo en verdad ente" es léxicamente una redundancia $y$, sin embargo, no podemos evitarlo, porque la propia condición de ente ha pasado a ser mediada, los criterios lo son de entidad, de qué es y qué no es ente, y a la vez son criterios, lo cual implica que algo (por lo tanto: ente) queda fuera. Esta notable situación comporta que lo en verdad ente esté obligado a manifestarse como precisamente todo lo ente; pero, cuando esto se haya cumplido, entonces se habrá hecho valer lo que ya estaba en el primer desdoblamiento, a saber: que negando lo "otro" se niega también lo "uno", y no hay ni lo uno ni lo otro; la historia que empieza con "en qué consiste el haber" termina en que simplemente no hay. Llegado este punto, la contraposición válida no es la que supuestamente habría entre el nihilismo y alguna otra cosa, sino la que hay en el propio fenómeno que llamamos nihilismo, a saber: nihilismo como mero estado de cosas nihílico frente a nihilismo como asunción (apropiación) de ese mismo estado de cosas. Esta contraposición es la que hay entre las figuras nietzscheanas del «último hombre» y el «transhombre», y esa misma oposición es la que constituye todo el proyecto de Nietzsche, el cual puede ser definido como el proyecto de la asunción del nihilismo. Así, las nociones clave del pensamiento de Nietzsche (como el eterno retorno y la voluntad de poder) pueden ser detalladamente interpretadas como intentos de diseñar un "andar con" las cosas que se atenga en efecto y radicalmente a la situación nihílica, pues se asume, por parte de Nietzsche, que esto es precisamente lo más difícil, lo que requiere un más largo y metódico esfuerzo, y lo es precisamente porque a la situación (nihílica) es inherente no reconocerse como tal ${ }^{4}$. Pues bien, el "no hay", la ausencia de contenidos vinculantes, significa que no estoy vinculado por nada y, en consecuencia, algo así como que hago lo que me da la gana, y en esto está incluido el que el "yo" aludido en esta misma fórmula, precisamente por su ausencia de vinculación, es cualquiera, de manera que el despliegue semántico de ese "hago lo que me da la gana" es que yo he de poder hacer todo aquello tal y bajo condiciones tales que el hecho de que yo lo haga no sea incompatible con que cualquier otro bajo las mismas condiciones

${ }^{4}$ A propósito de la posible interpretación del eterno retorno en esta línea, cf. mi artículo El tiempo del eterno retorno, en: Er, revista de filosofía, n. 14,1992 , pp. 99-109. 
pueda también, si quiere, hacerlo; que es la misma fórmula a la que anteriormente y por dos caminos habíamos llegado.

Nos hemos ocupado hasta aquí de señalar la capacidad esclarecedora que la concepción kantiana del derecho tiene en relación con otras ubicaciones del fenomeno "derecho" en pensadores de la modernidad. Para un correcto uso ulterior de esas conexiones será importante no perder de vista cierto carácter que lo kantiano aquí especialmente aludido comparte con todo lo demás de Kant, a saber, eso que tantas veces se ha recordado como "la finitud", es decir: el que la "forma" (las "condiciones de la posibilidad") no genera los contenidos; un concepto tan repetido en general como a menudo olvidado en el desarrollo de las cuestiones particulares, por ejemplo: cualquiera puede observar cómo al principio ético kantiano se le reprocha de vez en cuando su incapacidad para decirnos qué debemos hacer, con lo cual se olvida que jamás podría decírnoslo, pues no puede kantianamente tratarse de un principio de génesis de decisiones, sino que éstas en cualquier caso tienen lugar ya, y todo lo que puede haber es un principio de autocrítica de las mismas. De igual manera, el principio kantiano del derecho no podría jamás ser algo de lo cual, por así decir, debiesen deducirse las leyes de un Estado; se supone que éstas (o lo que pretende ser tal) se encuentran ahí contingentemente como resultado de luchas asimismo contingentes; el principio general del derecho sirve para someterlas a análisis crítico desde el punto de vista de en qué medida eso que hay está siendo en verdad un Estado.

Barcelona, noviembre de 2003 